\title{
Restricted Diachronic Composition and Special Relativity
}

\author{
Stephan Torre
}

\begin{abstract}
When do objects at different times compose a further object? This is the question of diachronic composition. The universalist answers 'under any conditions whatsoever'. Others argue for restrictions on diachronic composition: composition occurs only when certain conditions are met. Recently some philosophers have argued that restrictions on diachronic compositions are motivated by our best physical theories. In Persistence and Spacetime and elsewhere, Yuri Balashov argues that diachronic compositions are restricted in terms of causal connections between object stages. In a recent paper, Nick Effingham ([2011]) argues that the standard objections to views that endorse restrictions on composition do not apply to a view that restricts composition according to compliance with the laws of nature. On the face of it, such restrictions on diachronic composition preserve our common sense ontology while eliminating from it scientifically revisionary objects that travel faster than the speed of light.

I argue that these attempts to restrict diachronic composition by appealing to either causal or nomological constraints face insurmountable difficulties within the context of Special Relativity. I discuss how the universalist should best respond to Hudson's argument for superluminal objects and, in doing so, I present and defend a new sufficient condition for motion that does not entail that such objects are in superluminal motion.

$0 \quad$ Introduction

1 Diachronic Composition

$2 \quad$ Diachronic Composition and Superluminal Objects

$3 \quad$ Restricting Diachronic Composition

$4 \quad$ Causal and Nomological Restrictions on Composition in a Relativistic Context

$5 \quad$ Superluminal Objects and Motion

6 Conclusion
\end{abstract}

\section{Introduction}

Under what conditions do objects at different times compose an object? This is the question of diachronic composition. The universalist answers ‘under any conditions whatsoever’. Others argue for restrictions on diachronic composition: composition occurs only when certain conditions are met. Recently some philosophers have argued that restrictions on diachronic compositions are motivated by our best physical theories. In Persistence and Spacetime and elsewhere, Yuri Balashov argues that diachronic compositions are restricted in terms of causal connections between object stages. In a recent paper, Nikk Effingham ([2011]) argues that the standard objections to views that endorse restrictions on composition do not apply to a view that restricts composition according to compliance with the laws of nature. On the face of it, such restrictions on diachronic 
composition preserve our common sense ontology while eliminating from it scientifically revisionary objects that travel faster than the speed of light.

In what follows, I argue that these attempts to restrict diachronic composition by appealing to either causal or nomological constraints face insurmountable difficulties within the context of Special Relativity. In section 1, I begin by introducing the notion of diachronic composition and presenting the Diachronic Composition Question. In section 2, I present a version of Hud Hudson's argument for the conclusion that the four-dimensionalist who accepts universalism about composition is committed to the existence of superluminal objects, objects that move faster than the speed of light. In section 3, I present Balashov's reply which restricts composition by appealing to immanent causation and Effingham's reply which restricts composition by appealing to accordance with the laws of nature. I also outline some of the motivations for causal and nomological restrictions on diachronic composition. In section 4, I raise difficulties for both approaches within the context of Special Relativity. Finally, in section 5, I discuss how the universalist should best respond to Hudson's argument for superluminal objects and, in doing so, I present and defend a new sufficient condition for motion that does not entail that such objects are in superluminal motion.

\section{Diachronic Composition}

According to common sense, the four legs and the tabletop that my laptop is resting on compose a table. According to common sense, there is no object composed of all current Romanian kindergarteners, the first cigar Churchill ever smoked, and the southernmost Starbucks. These observations naturally lead to the following question: When do a bunch of objects make up or compose a further object and when do they fail to do so? More formally: what are the necessary and jointly sufficient conditions for a plurality of objects to compose a further object? This is Peter Van Inwagen’s well-known Special Composition Question (SCQ):

(SCQ) What necessary and jointly sufficient conditions must any xs satisfy in order for them to compose a further material object?

Nihilists claim that composition never occurs. ${ }^{1}$ There are no conditions that a group of objects can satisfy in order for them to compose a further material object. Whereas common sense alleges that the four legs and the tabletop compose a table, this is in fact not the case: nothing ever composes anything else. According to the nihilist, what the world really contains is just a collection of simples (objects without parts) that are arranged table-wise, chair-wise, person-wise, etc.

\footnotetext{
${ }^{1}$ Nihilism has been defended in (Rosen and Dorr [2002]; Unger, [1979]; Cameron [2010]) and Sider [forthcoming]).
} 
In stark contrast, the universalist claims that composition always occurs. The four legs and the tabletop compose a table and all current Romanian kindergarteners, Churchill's first cigar, and the southernmost Starbucks also compose a further object. In fact, for any plurality of objects that exists, there is an object that contains all and only those objects as parts. So, in addition to an ontology of tables, chairs and persons, the universalist is committed to an ontology containing innumerably-many scattered and gerrymandered objects.

Restricted theories of composition deny that composition always occurs and also deny that composition never occurs. Some versions of restricted composition maintain that there are stateable informative conditions under which composition occurs, such as if the parts are in contact with one another, if they are arranged so as to give rise to a living organism, or if they are arranged so as to give rise to a conscious being. ${ }^{2}$ Other restricted composition theorists, brutal compositionalists, grant that composition occurs sometimes, but not always, but deny that we can provide informative conditions under which composition occurs. ${ }^{3}$ Brutal compositionalists might grant that the four legs and tabletop compose a table, but deny that there are any state-able conditions for why they do. It seems that one of the main reasons for adopting a restricted theory of composition is that it best accords with common sense: the restricted compositionalist can maintain that tables, chairs, and persons are cases where composition occurs but composition fails to occur in the case of scattered or gerrymandered objects.

In this paper I wish to focus on a certain question concerning composition, namely when composition occurs across time. We can wonder whether the four table legs and the tabletop now compose a further object, namely a table. But we can also consider classes of objects at distinct times. Is there a single object that contains as parts the table at $1 \mathrm{pm}$ and the table at $2 \mathrm{pm}$ ? Is there a single object, the Obameron, composed of Barak Obama on Mondays, Wednesdays and Fridays and David Cameron on Sundays, Tuesdays, Thursdays, and Saturdays? Common sense says that there is an object of the first sort, but not an object of the second sort.

The two main competing theories about the nature of persistence, endurantism and perdurantism, would provide different descriptions of what is being asked in the above questions. The endurantist maintains that objects are wholly present at each moment at which they exist. So the endurantist would state the above questions more perspicuously as follows: Is there one object that is the wholly present table at $1 \mathrm{pm}$ and the wholly present table at $2 \mathrm{pm}$ ? Is there a single object that is the wholly present Barack Obama on Mondays, Wednesdays and Fridays and the wholly present David Cameron on Sundays, Tuesdays, Thursdays and Saturdays?

\footnotetext{
${ }^{2}$ The view that composition only occurs in the case of living organisms is defended in (Van Inwagen [1990]) and the view that composition only occurs in the case of conscious beings is defended in (Merricks [2001]).

${ }^{3}$ For a defense of brutal composition see (Markosian [1998]).
} 
The perdurantist denies that objects exist at multiple times by being wholly present at various times. The perdurantist claims that objects exist at multiple times by having distinct temporal parts that exist at those times. Just as we think that the table exists at multiple points in space by having distinct spatial parts that exist at those points, the perdurantist thinks that the table exists at multiple times by having a 1:00 part, a 1:01 part, a 1:02 part, etc. So the perdurantist would state the above questions more perspicuously as follows: Is there one object that has the 1pm table temporal part and also the 2pm table temporal part? Is there a single object that is composed of Barack Obama’s Monday, Wednesday, Friday temporal parts and David Cameron’s Sunday, Tuesday, Thursday, Saturday temporal parts?

I will follow Ted Sider ([2001]) by framing the question in a way that is amenable to both an endurantist treatment of persistence and a perdurantist treatment. Following Sider, let us call an 'assignment' "any (possibly partial) function that takes one or more times as arguments and assigns non-empty classes of objects that exist at those times as values; and let us say that an object $\mathrm{x}$ is a diachronic fusion ('D-fusion', for short) of an assignment $f$ iff for every $t$ in $f$ 's domain, $\mathrm{x}$ is a fusion-at-t of $f(\mathrm{t})$ )" (Sider [2001], p. 133).

To apply Sider's notion of a diachronic fusion to the case of the table, let us take $f_{\mathrm{t}}$ to be an assignment that assigns, to each time, $\mathrm{t}$, in the life of the table, the collection consisting of the legs of the table at $\mathrm{t}$ and the tabletop at $\mathrm{t}$. Let us call the collection of objects that is assigned to each time, $\mathrm{t}$, TableParts . There is a diachronic fusion of $f_{\mathrm{t}}$ just in case there exists an object, o, such that for every $\mathrm{t}$ in $f_{\mathrm{t}}$ 's domain, $\mathrm{o}$ is the fusion-at-t of TableParts $\mathrm{s}_{\mathrm{t}}$.

We can frame the question about when diachronic composition occurs as a question about when a given assignment has a diachronic fusion. Let us call this question the Diachronic Composition Question or (DCQ):

(DCQ) What necessary and jointly sufficient conditions must a given assignment, $f$, satisfy in order for there to be a diachronic fusion of $f$ ?

It is this question, rather than SCQ, that will be the focus of the rest of the paper.

\section{Diachronic Composition and Superluminal Objects}

In ([2002]) Hud Hudson argues that if we accept certain widely held philosophical doctrines and an uncontroversial assumption about motion, then we should grant that there are objects that move faster than the speed of light. In fact, if we accept such doctrines, then we should grant that, for any multiple of the speed of light, there exists an object that travels in continuous motion at that 
speed. Two of the widely held philosophical doctrines that lead to Hudson's conclusion are a metaphysics of temporal parts and universalism about composition. ${ }^{4}$

Since we will be considering Hudson's argument within the context of Special Relativity, it will be helpful to briefly discuss some of the fundamental relations of relativistic spacetime.

\section{Figure 1: Some Basic Features of Relativistic Spacetime}

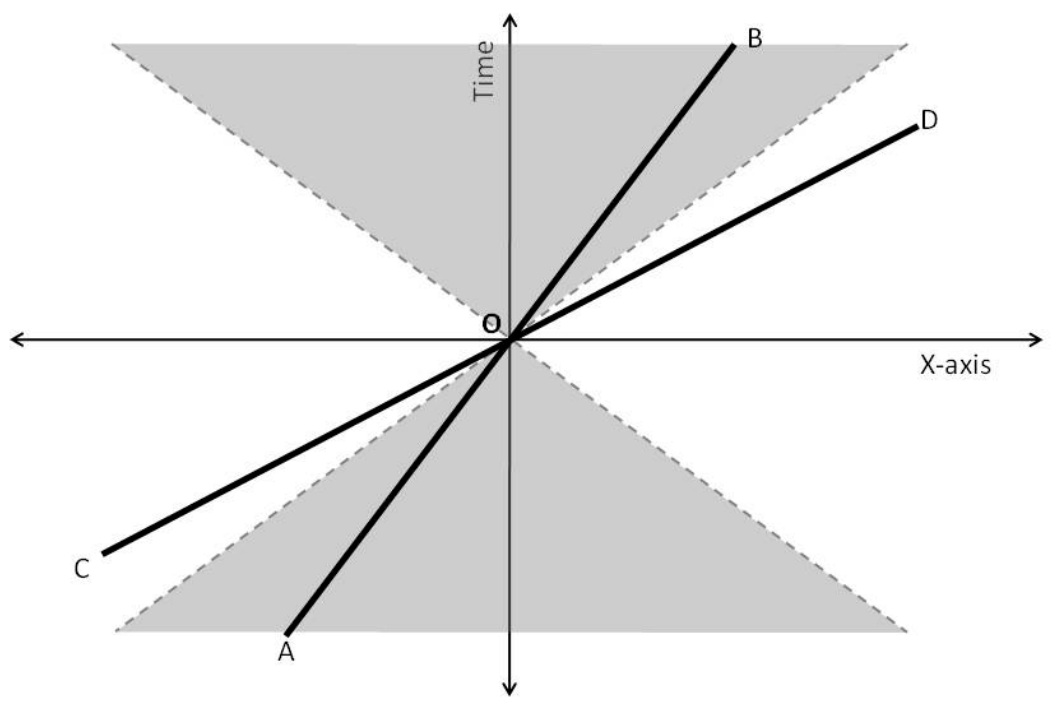

Figure 1 is a spacetime diagram in which the vertical axis represents the temporal dimension and the horizontal axis represents one spatial dimension. Let us take our axes to be centered on an event within spacetime, o. The coordinates in our graph are chosen in such a way that an object that is initially at rest remains at rest, and an object that is initially in motion continues its motion without a change in speed or direction. This provides us with an inertial frame of reference. Suppose a flash of light is emitted from o. The dashed lines above the $\mathrm{x}$-axis represent the path the photons would travel. The dashed lines below the $\mathrm{x}$-axis represent those spacetime points such that, if a flash of light were emitted from them, the photons would reach o. The shaded region above the xaxis represents the future lightcone of o and the shaded region below the $\mathrm{x}$-axis represents the past lightcone of o. The regions inside the future lightcone of o and the past lightcone of o represent those spacetime points that are timelike separated from o. For any spacetime point, p, in the future lightcone of o, it is possible for a causal signal originating at o to reach p. For any spacetime point, $\mathrm{p}$, in the past lightcone of $\mathrm{o}$, it is possible for a causal signal originating at $\mathrm{p}$ to reach $\mathrm{o}$. Those regions outside of the shaded areas represent those regions of spacetime that are spacelike separated

\footnotetext{
${ }^{4}$ Other assumptions that Hudson relies on are the Doctrine of Arbitrary Undetached Parts and what is taken to be an uncontroversial assumption about motion. I introduce the assumption about motion that Hudson's argument relies on below.
} 
from o. According to relativistic physics, no causal signal originating at o can reach any of the points in this region nor can a causal signal originating from any points in this region reach o.

A worldline is the path of an object through spacetime. In Figure 1, the line AB represents the worldline of an object traveling at constant velocity relative to o. As the figure represents, this object is traveling at a speed slower than the speed of light since its worldline falls inside the past lightcone of $\mathrm{o}$ and inside the future lightcone of o. The line CD represents the worldline of an object with a superluminal velocity: it travels faster than the speed of light, as is represented by the fact that its worldline lies outside the past and future lightcones of o.

In what follows, I will outline Yuri Balashov’s presentation of Hudson's argument.

Balashov asks us to consider a Flatland world in which objects at a time are extended in at most two spatial dimensions. Furthermore, let us suppose a metaphysics of temporal parts: objects in Flatland persist through time by having two-dimensional parts located at each time at which they exist.

\section{Figure 2: Box from Thread's Rest-Frame}

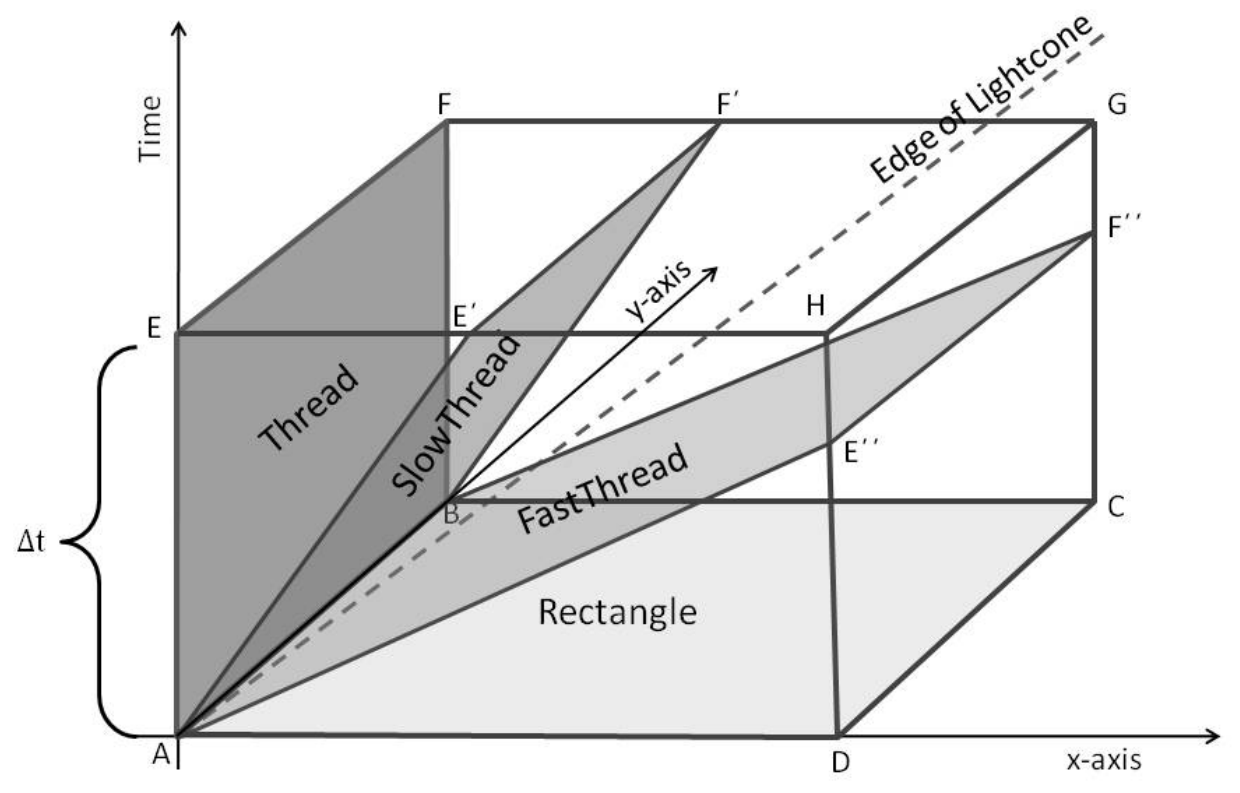

Figure 2 represents a persisting object, Box, in Flatland. The x- and y-axes represent the two spatial dimensions of Flatland and the vertical t-axis represents the temporal dimension of Flatland. In Figure 2, Box is marked out by ABCDEFGH. Box is an object that persists throughout the temporal interval $\Delta \mathrm{t}$. Box has a temporal part at each moment throughout $\Delta \mathrm{t}$. Its first temporal part, Rectangle, is marked out by ABCD in Figure 2. Whereas Box persists throughout the interval $\Delta \mathrm{t}$, Rectangle exists only at an instant. Consider Thread, marked out by ABEF. Thread also has temporal parts at each moment throughout the interval $\Delta \mathrm{t}$. AB is the first temporal part of Thread. 
Whereas Box occupies a three-dimensional box throughout its career, Thread occupies a twodimensional rectangle. Let us call the vertical cross-sections of ABCDEFGH (those orthogonal to the $\mathrm{x}$-axis) ' $\mathrm{x}$-slices'. Thread is an $\mathrm{x}$-slice of Box.

What is it for an object to be in motion relative to a given inertial frame? Hudson and Balashov both accept the following "relatively innocuous” assumption about motion:

(MO): Necessarily (a material object, $\mathrm{x}$, is in motion during an extended interval $\mathrm{t}$ in inertial frame f, if (i) at every instant in t, x occupies a region of space, and (ii) at no two instants in $t$ does $x$ occupy the same region of space) (Hudson [2003], p. 16). ${ }^{5}$

An object that occupies the same region of space throughout a temporal interval relative to a given frame of reference is represented by having a vertical worldline: it occupies the same position on the $\mathrm{x}$ - and $\mathrm{y}$-axis throughout the given interval and so fails to be in motion. An object in motion throughout a temporal interval relative to a given frame of reference is represented by a non-vertical worldline: it occupies different positions on either the $\mathrm{x}$ - or the $\mathrm{y}$-axis throughout the given interval. Thread is not in motion relative to this frame of reference since it occupies the same region of space throughout its career.

According to Hudson, there are a number of other objects that have $\mathrm{AB}$ as their initial temporal part. One such object is SlowThread (marked by $A B E^{\prime} F^{\prime}$ on the graph). SlowThread is made up of distinct temporal parts of x-slices of Box. In fact, no two temporal parts that compose SlowThread are part of the same x-slice. Furthermore, any two distinct temporal parts that compose SlowThread are timelike separated. According to (MO), SlowThread is in motion throughout the interval $\Delta \mathrm{t}$ relative to this frame of reference. Furthermore, SlowThread is in subluminal motion (it travels slower than the speed of light) since its path is within the future lightcone of AB.

Next consider FastThread (marked by ABE" ${ }^{\prime \prime}$ on the graph). Like SlowThread, FastThread is composed of temporal parts of x-slices of Box. However, unlike SlowThread, the temporal parts that compose FastThread are spacelike separated from one another. According to (MO), FastThread is in motion throughout the interval $\Delta \mathrm{t}$ since it occupies a different location in space at each moment throughout $\Delta \mathrm{t}$. Furthermore, FastThread is in superluminal motion (it travels faster than the speed of light) since its path is outside of the future lightcone of $A B$.

If we accept the reasoning that has led us to the conclusion that FastThread is in superluminal motion, then it is easy to see that we should grant that, for any multiple of the speed of

\footnotetext{
${ }^{5}$ I have added 'in inertial frame f' to Hudson's original formulation in order to make it explicit that the sufficient condition for motion is frame relative.
} 
light we wish to specify, there is some object that travels at that speed. There is some set of temporal parts of distinct x-slices of Box that compose any such object.

\section{Restricting Diachronic Composition}

It certainly seems surprising that, if we begin with a few assumptions that are commonly held by metaphysicians: universalism about composition, a metaphysics of temporal parts, and the standard account of motion, we reach the conclusion that there are many objects travelling many times faster than the speed of light. This conclusion is so surprising that some have taken this to be a demonstration of the falsity of one or more of the commonly held assumptions. This is Balashov's response. Balashov accepts Hudson's conditional claim, namely that if universalism is true, the doctrine of temporal parts is true, and the standard account of motion is true, then there are many objects that travel many times faster than the speed of light. But Balashov takes the truth of this conditional to support the falsity of one of the conjuncts in the antecedent. He states, "I believe that here we have one of those cases where a sobering modus tollens is more plausible than a sweeping modus ponens" (Balashov [2003a], p. 7).

The culprit, according to Balashov, is universalism about composition. Balashov chooses to restrict diachronic composition. According to Balashov, not all objects that exist at distinct moments compose a further object. He states the restriction in the following passage:

What binds a series of temporal parts of Thread into a robust material object is not just spatiotemporal continuity but a stronger relation of a broadly causal sort. Following W.E. Johnson and David Armstrong, one could call it immanent causality, a form of causality confined to a single perduring particular and welding its temporal parts together 'to constitute the single thing that exists through time' (Armstrong [1997], p. 74)...the presence of such a relation makes a series of temporal parts of a robust object different in kind from a juxtaposition of its contemporaneous spatial parts, such as the sum of cross-sectional parts of ABCD. A mark put on a temporal part persists through later parts, whereas a mark in some spatial part does not persist through space...(Balashov [2003a], p. 9)

Balashov chooses to restrict diachronic composition to those parts that are related by immanent causation. What exactly is immanent causation? Answering this question turns out to be quite difficult. ${ }^{6}$ Balashov attempts to clarify the notion by an object's ability to transmit a mark from early stages to later stages. If I spray some red paint on this table at $1 \mathrm{pm}$, the later stages of the table, such as its 2pm stage, will also be red in the same place. Whereas if I spray some red

\footnotetext{
${ }^{6}$ See (Zimmerman [1997]) for one such attempt.
} 
paint on this table at $1 \mathrm{pm}$, the $2 \mathrm{pm}$ stages of the table next to it will not be red. So it seems that the stages of this table are tied together in an objective, physical way in which the $1 \mathrm{pm}$ stage of the table and the 2pm stages of the neighboring table or the Eiffel Tower are not.

A proposal similar to Balashov’s is given by Nikk Effingham ([2011]) in “Undermining Motivations for Universalism”. Rather than appealing to immanent causation as a restriction on diachronic composition, Effingham proposes a nomological restriction. Although Hudson accepts the conclusion of his argument and accepts that there are many material objects that travel many times faster than the speed of light, he claims that there is a sub-class of material objects, the proper continuants, to which the laws of nature apply. Effingham claims that anyone who adopts Hudson's distinction between material objects and proper continuants will have to answer the following question:

Proper Continuant Question (PCQ): What are the jointly necessary and sufficient conditions some ys that compose an object must meet for it to be the case that they compose a proper continuant? (Effingham [2011], p. 703).

Effingham labels the answer to PCQ ' $\mathrm{X}$ ' and he goes on to note that the answer that one gives to PCQ can also be given as an answer to SCQ. Effingham states, "Call Xism the answer to the SCQ you get if you do use $\mathrm{X}$ as an answer to the SCQ rather than the PCQ. So if X was that the $y$ s must meet condition F to be proper continuants, then Xism is the claim that the ys must meet condition F in order to compose a further object” (Effingham [2011], p. 704). As Effingham notes, Xism will be a restricted answer to SCQ. Since X is an answer to PCQ and for an object to be a proper continuant is for the laws of nature to apply to it, Xism restricts composition to only those objects to which the laws of nature apply. One of the laws of nature, Effingham claims, is the speed constraint. Effingham states the speed constraint as the claim that there exist no objects that travel faster than the speed of light. Xism rules out superluminal objects on the basis that such candidate objects violate the speed constraint. Effingham argues that many of the arguments that the universalist wields against restricted theories of composition fail to apply to Xism.

Appealing to nomological or causal considerations as a means of restricting diachronic composition appears to have a number of benefits. At first appearances, it seems to accord with common sense: why do the various stages of this table compose an object, whereas the alternating day stages of Barack Obama and David Cameron fail to compose an object? A plausible answer seems to be because the stages making up the first set are immanently causally related; the right kind of causal relations obtain between its parts, but the stages in the second group fail to stand in 
the appropriate causal relation. Whereas a scratch on the table on Tuesday is transmitted to the table on Wednesday, if Obama bruises himself on Monday, the mark will not be transmitted to Cameron on Tuesday.

Furthermore, if we allow any haphazard assignment of object stages to count as a further object, we get what seems like a scientifically revisionary conclusion: namely that for any multiple of the speed of light we care to specify, there is some object that travels at that speed. It seems that good metaphysics should be in accord with our best physics and our best physics maintains that nothing travels faster than the speed of light. Since causal signals are always propagated at subluminal speeds, adopting a causal restriction on composition ensures that fast moving objects of the sort that Hudson appeals to will not figure into our ontology. Similarly, if we restrict composition to only those objects that obey the laws of nature, and our best physics maintains that the speed constraint is such a law, then our ontology will fail to include superluminal objects. In what follows, I will argue that, despite these apparent benefits, we should resist the offer to adopt either causal or nomological restrictions on composition.

\section{Causal and Nomological Restrictions on Composition in a Relativistic Context}

As we saw in section 2, Balashov suggests immanent causation as a means of keeping objects like SlowThread and FastThread out of one's ontology. Requiring immanent causation as a necessary condition for diachronic composition succeeds in ruling out superluminal objects. However, I will argue that positing such a restriction leads to difficulties within the context of Special Relativity.

In Persistence and Spacetime, Balashov claims that one can adopt immanent causation as a necessary condition for diachronic composition, but such a restriction is independent of how one treats synchronic composition. Balashov states it as follows:

The 4Dist is free to treat synchronic and diachronic composition differently. The latter may be causally grounded in a way the former is not. Even in the absence of any principled criterion of restricted composition at a time, the 4Dist can draw a distinction between series of object stages cemented by a broadly causal relation and those that are not. Thus defending some version of restricted synchronic composition is not a prerequisite for resisting diachronic universalism. Accordingly, there is no pressure for the 4Dist to be a universalist across the board. She is free to banish, not merely disregard, loose collections of unrelated temporal parts...even if she is not yet prepared to do away with loose collections of spatial parts. 
While some may view such partial rejection of universalism as arbitrary, I submit that it simply follows the joints of nature (Balashov [2010], p. 88).

However, combining immanent causation as a necessary condition on diachronic composition with almost any theory of synchronic composition leads to problems within the context of Special Relativity.

A natural way of stating the restriction on diachronic composition using Sider's notion of assignments is as follows:

(DFIC) An object $\mathrm{x}$ is a diachronic fusion of a given assignment $f$ only if, for any distinct times, $\mathrm{t}_{1}$ and $\mathrm{t}_{2}$, in the domain of $f, f\left(\mathrm{t}_{1}\right)$ is immanently causally related to $f\left(\mathrm{t}_{2}\right)$.

Let us return to Figure 2. Recall that according to Balashov, Thread is an object because its parts stand in the requisite causal relation, but SlowThread and FastThread are not objects because their candidate parts fail to stand in the requisite causal relation. Suppose we were to adopt a view of synchronic composition according to which Rectangle counts as an object. Presumably, Rectangle, and its real world, three-dimensional counterpart, will count as an object on most theories of synchronic composition: universalism, certain versions of brutal composition, accounts that appeal to contact, etc. Nihilism will be one view on which Rectangle and its three-dimensional counterpart fail to count as an object.

However, one of the key implications of Special Relativity is that the notion of synchrony is relative to an inertial frame: there is no frame-independent fact of the matter about whether two spacelike separated events in spacetime are synchronous. So if we adopt a theory of composition that entails that Rectangle is an object, there will be frames of reference according to which the parts that comprise Rectangle are not synchronous. Consider the frame of reference 'SlowThreadRest' according to which the (candidate) temporal parts for SlowThread are such that they trace out what would be an object at rest (see Figure 3). 


\section{Figure 3: Box from SlowThread's Rest Frame}

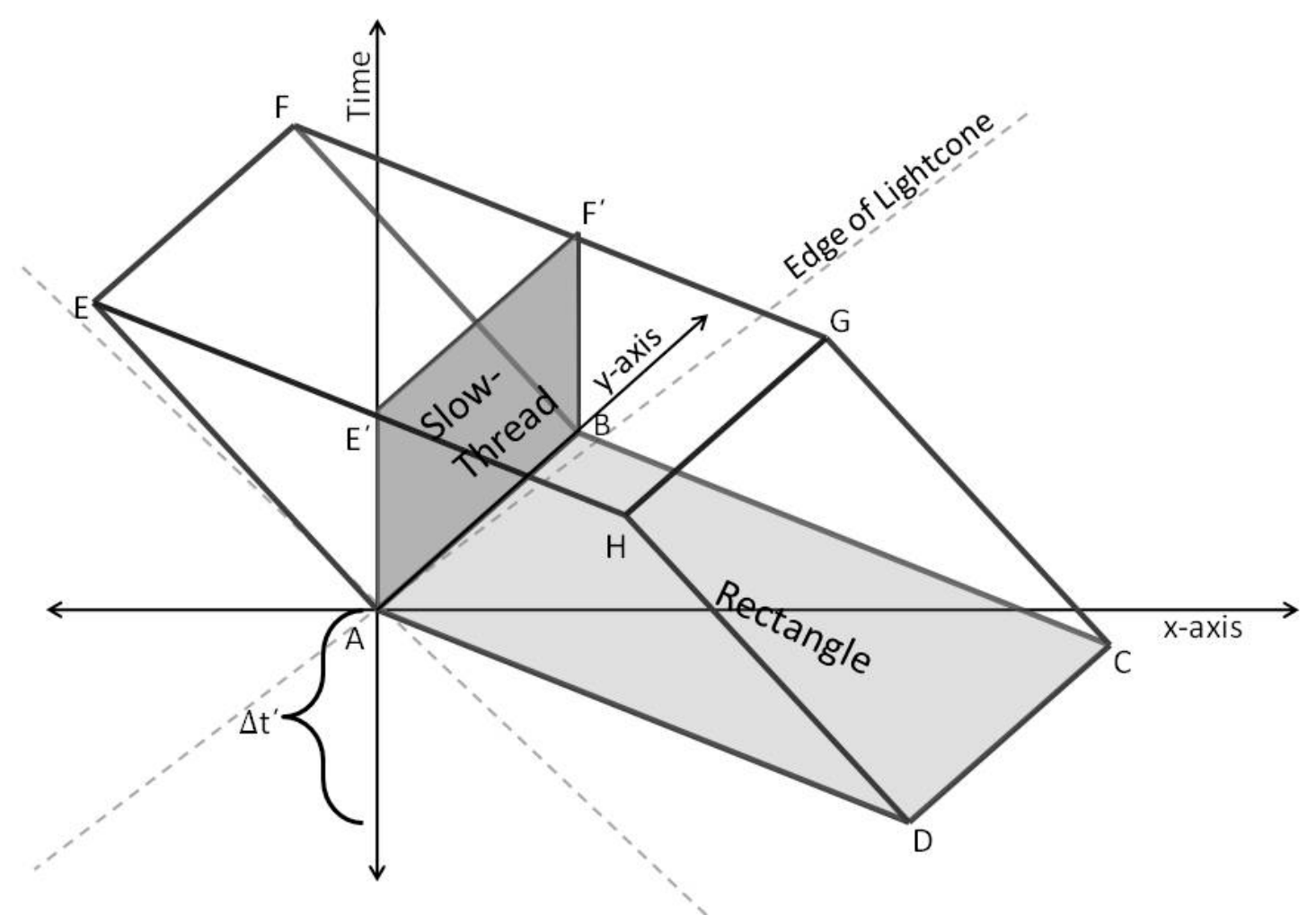

Relative to SlowThread-Rest, the parts of Rectangle fail to be synchronous. Rather they are spread out throughout the interval $\Delta \mathrm{t}^{\prime}$. So, at least relative to SlowThread-Rest, Rectangle fails to be an object according to (DFIC): Consider the assignment $f_{\text {rectangle }}$ that assigns to each time, $\mathrm{t}$, in the interval $\Delta \mathrm{t}^{\prime}$ the candidate parts of Rectangle that exists at $\mathrm{t}$. For any two distinct times, $\mathrm{t}_{1}$ and $\mathrm{t}_{2}$, in the domain of $f_{\text {rectangle, }}, f_{\text {rectangle }}\left(\mathrm{t}_{1}\right)$ and $f_{\text {rectangle }}\left(\mathrm{t}_{2}\right)$ fail to be immanently causally related. That they fail to be immanently causally related is clear from the fact that the candidate parts that make up Rectangle are spacelike separated and so no causal signal can reach one event from any other. So if we grant an account of synchronic composition that entails that Rectangle is an object in ThreadRest (the inertial frame according to which Thread is at rest, see Figure 2), we get the following difficulty: Assume Rectangle is an object in Thread-Rest. If Rectangle is an object in Thread-Rest, then Rectangle is an object in SlowThread-Rest (presumably composition is not something that is frame-relative. I address the possibility of denying this in more detail below). But according to (DFIC) Rectangle is not an object in SlowThread-Rest.

An obvious reply suggests itself. The defender of immanent causation as a restriction on diachronic composition should not take the restriction to apply to any objects that fail to be synchronous, but rather to objects that are timelike separated. In "Restricted Diachronic Composition, Immanent Causality, and Objecthood: A Reply to Hudson” Balashov proposes an account of diachronic composition that applies only to timelike separated objects. In what follows I 
will consider whether such an account of diachronic composition can avoid difficulties when considering whether there exist composite objects like Rectangle. Balashov formulates the restriction not in terms of the frame-relative notion of objects existing at different times, but rather in terms of the frame-invariant notion of objects that are timelike separated. He distinguishes between two kinds of composition, diachronic composition 'composition ${ }^{\mathrm{D} \text { ' }}$ and synchronic composition 'composition's, He states that "Diachronic composition requires pairwise timelike or lightlike separation of the $x$ s and synchronic composition their pairwise spacelike separation...This is not to deny the univocal character of composition but only to distinguish two principle natural kinds of composition, which are mutually exclusive” (Balashov [2003b], p. 24). He then goes on to state the restriction on diachronic composition as follows:

(R) Objects that are not connected by immanent causality do not compose ${ }^{\mathrm{D}}$ anything; they also do not compose anything unless they belong to the same moment of time in the rest frame of their center of mass, in which case they may or may not compose something (by composing ${ }^{\mathrm{S}}$ it), depending on one's theory of synchronic composition (Balashov [2003b], p. 25).

It is worthwhile to spend some time formulating Balashov's proposed restriction precisely and distinguishing it from (DFIC) above. Note that up until now, we have been framing the question of diachronic composition in terms of when objects at distinct times compose a further object. However the notion of distinct times is a frame-relative notion. Two events may occur at different positions on the t-axis in Thread-Rest but fail to occur at different positions on the t-axis in another frame of reference. In order to properly formulate Balashov's proposed restriction, we must appeal to timelike separated spacetime regions rather than distinct times. Let us say that two regions of spacetime, R1 and R2, are spacelike separated just in case, for all spacetime points, p1 and p2, if p1 is in R1 and p2 is in R2, then p1 is spacelike separated from p2. Two regions of spacetime, R1 and $\mathrm{R} 2$, are timelike separated just in case, for all spacetime points, $\mathrm{p} 1$ and $\mathrm{p} 2$, if $\mathrm{p} 1$ is in $\mathrm{R} 1$ and $\mathrm{p} 2$ is in $\mathrm{R} 2$, then $\mathrm{p} 1$ is timelike separated from $\mathrm{p} 2$. Let us say that two objects, $\mathrm{x}$ and $\mathrm{y}$, are spacelike separated just in case they exactly occupy spacelike separated regions of spacetime. ${ }^{7}$ Similarly, we can say that two objects, $x$ and $y$, are timelike separated just in case they exactly occupy timelike separated regions of spacetime. The non-overlapping parts of Rectangle are spacelike separated since each part occupies a region of spacetime that is spacelike separated from the regions occupied

\footnotetext{
${ }^{7}$ Here I take 'exactly occupy' to be a locational predicate where an object o exactly occupies a region R just in case o has the same shape and size as $\mathrm{R}$ and o stands in all the same spatiotemporal relations as R. Adopting such a predicate is not without controversy. See (Gilmore [2008], esp. pp.1228-1229 and (Parsons [2007], pp. 201-32) for some further discussion.
} 
by Rectangle's other non-overlapping parts. As noted above, spacelike separation and timelike separation are frame-invariant relations: if an object's parts are spacelike (timelike) separated in one frame, they are spacelike (timelike) separated in all frames. ${ }^{8}$

We can now provide a different account of diachronic fusion from the account originally proposed by Sider. Rather than taking a given assignment, $f$, to be a function from times, t, to objects that exist at $\mathrm{t}$, let us take an assignment to be any (possibly partial) function that takes one or more timelike separated spacetime regions as arguments and assigns non-empty classes of objects that exist at those regions as values; and let us say that an object $\mathrm{x}$ is a timelike-separated fusion of an assignment $g$ iff for every timelike separated region, $\mathrm{R}$, in $g$ 's domain, $\mathrm{x}$ is a fusion-at-R of $g(\mathrm{R})$. We can now state Balashov's proposed restriction on timelike separated fusions as follows:

(TSIC) An object $\mathrm{x}$ is a timelike-separated fusion of a given assignment $g$ only if, for any timelike separated spacetime regions, $\mathrm{R}_{1}$ and $\mathrm{R}_{2}$, in the domain of $g, g\left(\mathrm{R}_{1}\right)$ is immanently causally related to $g\left(\mathrm{R}_{2}\right)$.

This revised view allows us to claim that, on some account of spacelike separated composition, the candidate parts of Rectangle compose an object and this fails to violate the necessary condition on timelike-separated composition since the parts of Rectangle are spacelike separated rather than timelike separated and so no causal constraint is necessary. Since being spacelike separated and being timelike separated are frame-invariant relations, we avoid the problematic conclusion reached earlier according to which granting synchronic composition in one frame leads to a violation of the necessary condition on diachronic composition in another frame.

However, adopting this notion of timelike separated composition and spacelike separated composition leads to a new difficulty. The original motivation for Balashov adopting immanent causation as a restriction on diachronic composition was to rule out superluminal objects like FastThread. Balashov explicitly grants that if FastThread exists, then FastThread is a superluminal object. But now the restriction on timelike separated composition in terms of immanent causation is silent with respect to superluminal objects. By definition, the restriction applies only to objects that are at subluminal speeds. Since there is no causal restriction with respect to composition for spacelike separated parts, there is nothing to rule out objects like FastThread. So, if one adopts the above restriction on composition in terms of timelike separation, one fails to banish from one's ontology the very objects that motivated the restriction in the first place.

\footnotetext{
${ }^{8}$ One may note that in introducing the notions of timelike separated parts and spacelike separated parts, we've left the notion of temporal parts behind. This is, I think, a move in the right direction. I am sympathetic to Gibson and Pooley's observation that the standard way of defining temporal parts is "a relic of the frame-relative perspective" and that "from a relativistic point of view, temporal parts are hardly worth defining” (Gibson and Pooley [2006], p. 163).
} 
A possible reply on the part of the proponent of (TSIC) who wishes to rule out objects like FastThread is to claim that only timelike separated fusions can properly be described as being in motion: motion doesn't apply to spacelike separated fusions and so Rectangle in SlowThread-Rest and FastThread in Thread-Rest fail to be in superluminal motion. But this obviously amounts to a rejection of (MO). The defender of immanent causation as a restriction on diachronic composition chose to banish superluminal objects by restricting composition rather than by rejecting (MO). But now he is rejecting both universalism and (MO). If rejecting (MO) is what is doing the work in ruling out superluminal objects, then the rejection of universalism appears unmotivated by considerations stemming from Hudson's argument. After all, the universalist could also choose to rule out superluminal objects by rejecting (MO).

How else might the defender of (DFIC) respond to the difficulties raised by considering objects like Rectangle? One particularly problematic response would be to say that Rectangle is an object relative to Thread-Rest but not an object relative to SlowThread-Rest; objects only exist relative to an inertial frame. According to this line of response, (DFIC) would be modified as follows:

(DFIC-R) An object $\mathrm{x}$ is a diachronic fusion of a given assignment $f$ relative to inertial frame $\mathrm{F}$ only if, for any distinct times, $\mathrm{t}_{1}$ and $\mathrm{t}_{2}$ relative to $\mathrm{F}$ in the domain of $f, f\left(\mathrm{t}_{1}\right)$ is immanently causally related to $f\left(\mathrm{t}_{2}\right)$.

This involves a relativization of ontology to frames of reference that is difficult to accept. On this view, what exists in the world changes as I press on the accelerator of my spaceship. If I am accelerating or if I am around for merely an instant, there seems to be no determinate answer to the question of what objects exist, since in either case, no single inertial frame is singled out. Furthermore, such a view seems to grant too much ontological authority to inertial frames. The standard way of thinking of inertial frames of reference is similar in many respects to how we would think of two different coordinate systems for spatial locations that differ in their choice of xand y-axes (suppose I assign spatial locations to a town by taking the southern city wall to lie on the $\mathrm{x}$-axis and you take Main Street to lie on the $\mathrm{x}$-axis). We take the two coordinate systems to describe and refer to the same underlying reality. Within Special Relativity inertial frames are construed in much the same way: they provide different ways of describing the same objects and events within Minkowsi spacetime. To claim that different domains of objects exist from one inertial frame to the next would involve a substantial departure from this picture. Such a move seems like an extreme conclusion to draw in response to the threat of superluminal objects, especially in light of the availability of more moderate alternatives. 
Related difficulties beset Effingham's proposal to restrict composition in terms of accordance with the laws of nature. Suppose we adopt Xism, the view that restricts composition to objects that are in accordance with the laws of nature. What should we say about Rectangle and its real world, three-dimensional counterparts? In order to focus our question a bit, let us consider whether a candidate object satisfies the speed constraint. Does Rectangle violate the speed constraint? Relative to SlowThread-Rest, Rectangle does exactly occupy the path of a candidate object in motion according to (MO): at every instant in $\Delta \mathrm{t}^{\prime}$, Rectangle occupies a region of space, and at no two instants in $\Delta \mathrm{t}^{\prime}$ does Rectangle occupy the same region of space (Figure 3). Furthermore, if we grant that Rectangle occupies the path of a candidate object in motion, then we must grant that the candidate object travels at superluminal speed since its trajectory lies outside the lightcone of A. So it seems that Rectangle does violate the speed constraint and so, according to Xism, Rectangle, and its actual world, three-dimensional counterparts, are not composite objects.

But if Xism entails that Rectangle is not an object in virtue of the fact that the candidate object violates the speed constraint, then it seems that something has gone wrong. Recall that in Thread-Rest, Rectangle is supposed to be the first temporal part of Box (Figure 2). If Rectangle is banished from the Xist's ontology on the grounds that it violates the speed constraint, then, by parity of reasoning, all later temporal parts of Box will also be banished. What holds true for Rectangle regarding the speed constraint will also hold true for Rectangle's real world, threedimensional counterparts: they too will exactly occupy the path of objects that travel at superluminal speeds relative to some frames of reference. So if the Xist banishes Rectangle from her ontology, it seems that she must also banish all spatially extended temporal parts from her ontology. All such parts will exactly occupy the path of a superluminal object relative to some frames of reference.

Effingham claims at the outset of his paper that he is considering the motivations for universalism "from a perdurantist point of view (where I'll take perdurantism to be the thesis that for every instant $t$ that an object exists at, it has an instantaneous temporal part at t)" (Effingham [2011], p. 696). But if temporal parts violate the speed constraint, then Xism is not an option for the perdurantist because it banishes the very objects that characterize the position. Given that Xism is not an option for the perdurantist, it is clear that Xism does not undermine the perdurantist's motivations for universalism.

\section{Superluminal Objects and Motion}

So what should we say about objects like FastThread? One popular line of response among advocates of unrestricted composition is to maintain that the dynamical laws only apply to a proper 
subset of material objects. As we have seen, Hud Hudson dubs those material objects to which the dynamical laws apply the 'proper continuants'. In a similar vein John Hawthorne advocates singling out "objects of interest to those formulating dynamical laws" which he calls "quality objects” from the rest which he calls “junk objects” (Hawthorne [2006], p. 111). Whereas an object like Box may count as a quality object and fall under the purview of the dynamical laws, these laws fail to apply to junk objects like FastThread. According to this line of response, the speed constraint only applies to a select group of material objects.

Although I am generally sympathetic to this kind of response, it does not succeed in resolving all the difficulties raised by considering material objects within the context of Special Relativity. We have already seen that restricting composition to the proper continuants (or, in Hawthorne’s terminology, “quality objects”) leads to difficulties when we consider one and the same object in different inertial frames. These difficulties arise from the fact that while a material object may fail to violate the speed constraint in one frame (such as Rectangle in ThreadRest) it may violate it in another (such as Rectangle in SlowThreadRest). This speaks against being able to draw a frame-independent distinction between quality objects and junk objects.

Furthermore, there is an underlying issue that is not addressed by restricting the application of the dynamical laws to a select group of material objects. Restricting the application of dynamical laws does not address whether (MO) provides a plausible sufficient condition for motion. Even if we maintain that the dynamical laws apply only to quality objects, (MO) nonetheless entails that many objects are in superluminal motion even though there seem to be good reasons for denying that such objects are properly described as being in motion. To see this, consider one of my temporal parts. Let us call my (instantaneous) 1pm temporal part in the rest frame of my 1pm temporal part's center of mass 'Body'. There are some frames of reference according to which the parts that make up Body exist at different times: relative to these frames of reference, the feetshaped parts of Body are not simultaneous with the head-shaped part of Body. Relative to these frames of reference, Body satisfies the sufficient condition for being in motion given by (MO). Furthermore, Body will count as an object in superluminal motion since the non-overlapping parts of Body are spacelike separated from each other.

The conclusion that Body is in superluminal motion relative to such frames of reference suggests that (MO) does not succeed in providing a sufficient condition for motion in any meaningful sense. It strikes me as highly revisionary to claim that persisting, spatially extended objects are made up of uncountably many superluminal objects: one for each temporal part. Even if we grant that the dynamical laws do not apply to such objects, (MO) commits us to maintaining that such objects are in superluminal motion. 
In a non-relativistic setting, the four dimensionalist is able to distinguish between the parts of an object that are spatially related, but fail to compose an object in motion (such as the feetshaped parts and head-shaped part of Body) and the parts of an object that compose an object in motion (such as my temporal parts throughout an interval in which I am walking). When we consider Body within the context of Special Relativity, a tension arises as to whether it counts as an object in motion. On the one hand, its non-overlapping parts are spacelike separated and this suggests that we ought to say what we said about parts that are merely spatially related in a nonrelativistic context: that they fail to compose an object in motion. On the other hand, our prerelativistic account of motion is one that involves change in spatial position throughout a temporal interval. The fact that, in the relativistic context, there are frames of reference according to which Body occupies different spatial coordinates throughout a temporal interval suggests that its parts do compose an object in motion in these frames of reference. The tension is resolved, I think, by considering which option does the least violence to our pre-relativistic concept of motion.

Granting that Body is not only in motion but in superluminal motion does more violence to our prerelativistic concept than granting that Body's parts, in virtue of being spacelike separated from one another, fail to compose an object in motion.

One might object by noting that if we embrace a universalist ontology, there are all kinds of objects that are highly revisionary in nature; such as the spatiotemporally scattered objects described in section 1. Given that the universalist is already comfortable with countenancing such objects and taking the proper subject of physics and much of our ordinary talk to concern only a proper subset of these objects, why not similarly grant that there are many more objects in motion than those that are dictated by common sense, some of which are in superluminal motion? ${ }^{9}$

I think the universalist is correct to claim that there are many more objects than those that are the proper subject of physics or dictated by common sense. The concept of being an object is sufficiently flexible that it can be correctly applied to gerrymandered and spatiotemporally scattered fusions of matter. But I deny that the concept of being an object in motion is so flexible as to be correctly applied to objects like Body that are made up of parts that are spacelike separated from one another. The universalist's comfort with having a plenitudinous ontology of objects, some of which are spatiotemporally scattered, does not, and should not, commit her to holding that fusions of spacelike separated parts compose objects in motion.

What relations between the parts of an object are sufficient for them to compose an object in motion? What's the relevant difference between the parts of Body, on the one hand, and my temporal parts throughout an interval in which I am walking, on the other hand? Reflecting on

\footnotetext{
${ }^{9}$ Thanks to an anonymous referee for pressing this objection.
} 
Body and its ilk suggests that motion does have a causal constraint. I propose rejecting (MO) and adopting of the following sufficient condition for motion:

(MOIC) Necessarily (a material object, $x$, is in motion during an extended interval $t$ in inertial frame $f$, if (i) at every instant in t, $x$ occupies a region of space, (ii) at no two instants in $t$ does $\mathrm{x}$ occupy the same region of space and (iii) for any two distinct instants in $\mathrm{t}, \mathrm{t} 1$ and $\mathrm{t} 2$, $\mathrm{x}$ at $\mathrm{t} 1$ is immanently causally related to $\mathrm{x}$ at $\mathrm{t} 2$ ).

It does not follow from (MOIC) that Body is in superluminal motion in any frame of reference since the non-overlapping parts of Body are not immanently causally related (this is clear from the fact that the non-overlapping parts of Body are spacelike separated). The same holds true for FastThread and Rectangle. The revised sufficient condition for motion avoids the unpalatable result that such objects are in superluminal motion.

I am somewhat uneasy in using the notion of immanent causation in (MOIC) since, as mentioned above, I find the very notion difficult to define and I also think that any account of immanent causation that applies to ordinary objects like people, cars and meteors will lead to cases where it is indeterminate or vague whether the parts of the object are immanently causally related. This, in turn, will entail that it is sometimes vague whether an object is properly described as being in motion. However, I think it is acceptable that vagueness crops up here. Sider convincingly argues that whether or not composition occurs cannot be a vague matter, and so this suggests that immanent causation is an unlikely candidate for restricting composition. But it seems unproblematic to grant that 'motion' is itself a vague term and that the appeal to 'immanent causation' in (MOIC) is one source of its vagueness.

Perhaps one could avoid including immanent causation as part of the sufficient condition for motion altogether by replacing (iii) with (iii*): for any two distinct instants in t, t1 and t2, $\mathrm{x}$ at $\mathrm{t} 1$ is timelike separated from to $\mathrm{x}$ at $\mathrm{t} 2 .{ }^{10}$ Modifying the sufficient condition for motion in this way would achieve the desired result of failing to entail that objects like Body and Rectangle are in superluminal motion. Perhaps this is a suitable alternative to (MOIC) but I have two reservations. Whether (MO) is best modified by requiring that the relevant parts are immanently causally related or by requiring that the relevant parts are timelike separated depends on whether one thinks objects like SlowThread are properly described as being in motion. I agree with Balashov's intuition that SlowThread ought not to count as an object in motion, however, unlike Balashov, I grant that SlowThread is an object. Second, I take the project of revising (MO) to be part of a more general project of giving the meaning of 'motion' within the context of Special Relativity. To that end, we

\footnotetext{
${ }^{10}$ Thanks to an anonymous referee for suggesting this alternative to (MOIC).
} 
should seek out jointly sufficient conditions for motion that are individually necessary. However it seems implausible that having timelike separated parts is necessary for motion. If it were, we could rule out superluminal objects from the armchair. Rather it is preferable to allow that there might be such objects in virtue of having parts that are related by superluminal immanent causation.

\section{Conclusion}

In Persistence and Spacetime, Balashov claims, "It is the universalist who has to convince the opponent that the opponent's appeal to firmly established results of science... is unmotivated" (Balashov [2010], p. 84). I have attempted to do exactly that. I have argued that both a causal constraint on composition in terms of immanent causation and a constraint in terms of accordance with the laws of nature both lead to significant difficulties when considering objects within the context of Special Relativity. I have also argued that considerations from Special Relativity motivate a modification the sufficient condition for motion. By including immanent causation as part of the sufficient condition for motion, we avoid the revisionary conclusion that there are many objects travelling many times faster than the speed of light without imposing problematic restrictions on composition. ${ }^{11}$

\section{Acknowledgements}

In writing this article, I received support from the Spanish Ministry of Science and Innovation's project Philosophy of Perspectival Thoughts and Facts (PERSP), CONSOLIDERINGENIO 2010 CSD2009-00056. I would also like to thank Nikk Effingham, Oliver Pooley and two anonymous referees for very helpful feedback on earlier drafts. 


\section{Works Cited}

Armstrong, D. [1980]: 'Identity Through Time'. in P. Inwagen (ed.), Time and Cause: Essays Presented to Richard Taylor. Reidel.

Balashov, Y. [2003a]: ‘Temporal Parts and Superluminal Motion’. Philosophical Papers 32, pp. 113.

Balashov, Y. [2003b]: 'Restricted Diachronic Composition, Immanent Causality and Objecthood: A Reply to Hudson’. Philosophical Papers 32, pp. 23-30.

Balashov, Y. [2005]: 'On Vagueness, 4D and Diachronic Universalism' Australasian Journal of Philosophy, 83, pp. 523-531.

Balashov, Y. [2010]: Persistence and Spacetime, Oxford: Oxford University Press.

Cameron, R. [2010]: ‘Quantification, Naturalness and Ontology’ in Allan Hazlett (ed.), New Waves in Metaphysics. Basingstoke: Palgrave Macmillan.

Dorr, C. and Rosen, G. [2002]: 'Composition as a Fiction', in R. Gale (ed.), The Blackwell Guide to Metaphysics, Oxford: Blackwell, pp. 151-174.

Effingham, N. [2009]: ‘Universalism, Vagueness and Supersubstantivalism’ Australasian Journal of Philosophy 87 (1), pp. $35-42$.

Effingham, Nikk [2011]: 'Undermining Motivations for Universalism’ Noûs 45, pp. 696-713.

Gibson, I. and Pooley, O. [2006]: 'Relativistic Persistence’ Philosophical Perspectives, vol. 20, pp.157-198.

Gilmore, C. [2008]: 'Persistence and Location in Relativistic Spacetime’ Philosophy Compass 3/6, pp. 1224-1254

Hawthorne, J. [2006]: 'Motion and Plenitude' in his Metaphysical Essays, Oxford: Oxford University Press, pp.111-143. 
Hudson, H. [2002]: 'Moving Faster than Light'. Analysis 62, pp. 203-5.

Hudson, H. [2003]: 'Immanent Causality and Diachronic Composition: A Reply to Balashov'. Philosophical Papers 32, pp. 15-22.

Lewis, D. [1986]: On the Plurality of Worlds, Oxford: Blackwell.

Markosian, N. [1998]: ‘Brutal Composition’, Philosophical Studies 92: pp. 211-249.

Merricks, T. [2001]: Objects and Persons, Oxford: Oxford University Press.

Parsons, J. [2007]: ‘Theories of Location’. Oxford Studies in Metaphysics 3, pp. 201-32.

Sider, T. [2001]: Four-Dimensionalism, Oxford: Clarendon Press.

Sider, T. 'Against Parthood'. Forthcoming in K. Bennett and D. Zimmerman, eds., Oxford Studies in Metaphysics, Volume 8, Oxford: Oxford University Press, 2013.

Unger, P. [1979]: ‘There Are No Ordinary Things’ Synthese 41, pp. 117-54.

Van Inwagen, P. [1990]: Material Beings, Ithaca (NY): Cornell University Press.

Zimmerman, D. [1997]: 'Immanent Causation’ Philosophical Perspectives, 11, Mind, Causation and World, pp.433-471. 